\title{
Codebook metrics evaluation for millimeter wave communications by antenna array response and signal to noise ratio
}

\author{
Adam Mohamed Ahmed Abdo ${ }^{1, *}$, Xiongwen Zhao ${ }^{1 *}$, Rui Zhang ${ }^{2}$, and Yu Zhang ${ }^{1}$ \\ ${ }^{1}$ School of Electrical and Electronic Engineering, North China Electric Power University, Beijing \\ 102206, China \\ ${ }^{2}$ National Key Laboratory of Electromagnetic Environment, China Research Institute of Radiowave \\ Propagation, Qingdao 266107, China
}

\begin{abstract}
Codebook design is a key point in beam-forming which is necessary for millimeter Wave (mmWave) communications to compensate its huge path loss. In our study, we demonstrate the benefits of the number of antenna elements for beamforming; we focus on the number of required beams to cover the whole circle with considering the available number of antenna elements. In addition to, we propose a new codebook structure based on unique phase states. Based on the Array Factor (AF) and Signalto-Noise Ratio (SNR), the proposed codebook is compared and evaluated with the uniform weighting codebook, circular array codebook, and IEEE802.15.3c standard codebook. The results show that the proposed codebook achieves SNR values and side-lobes cancel a bit better than IEEE 802.15.3c, but higher peak and wider dynamic range of SNR and better array directivity compared with the uniform weighting and circular antenna codebooks. Thus, the proposed codebook is expected to improve signal quality and increases user throughput significantly.
\end{abstract}

\section{Introduction}

The demand growth for wideband data transmission, $\mathrm{mmW}$ communication band, is getting considerable attention in wireless local area networks (WLANs) and wireless personal area networks (W-PANs), and in dense user scenarios for fifth generation (5G) radio systems. mmWavesystem is used in short rangeand high data rate communication system with huge unlicensed bandwidth, significant capacity and flexibility. Also, it allows high transmit power and less interference effect. Moreover, it can be applied in large file transfer and uncompressed high-definition video streaming [1]. Further improving transmission range of mmW band also enhances spatial efficiency [2], [3].

However, the mmWave band faces many impairments such as high free space path loss level, high attenuation, and oxygen absorption compared with low-frequency communication band [1], [3]. To compensate the significant path loss, the directional

\footnotetext{
${ }^{1}$ Corresponding author:adam_ma83@yahoo.com, zhaoxw@ncepu.edu.cn
} 
antenna is used to generate narrow directional beams, which provides significant gain [4], $[5]$.

Beam-forming techniques help to investigate many benefits including the system throughput enhancement, spatial reuse, and minimal interference. Moreover, as the antenna size is directly proportional to wavelength, the mmWave antenna is tiny compared with other low-frequency communication. Therefore, the beam-forming technology can be used to improve the link quality as well as angle information distribution. All these factors motivate the researchers to focus on mmW communication [4], [5]. Analog beam-forming is much less hardware complexity and power consumption constraint so that it preferred in $60 \mathrm{GHz}$ indoor mmWave communication [2], [5]. The main purpose of the array antenna is to improve the SNR by adding correlated signals and uncorrelated noise. This improvement measures regarding array gain or array response [8]. The mmWave communication uses two techniques. The first is codebook based beam steering to solve energy constraint. The second one has phased array antennas with fixed amplitude beam weighting vector to solve complexity problem. In phased array antenna, to generate the beam pattern by shifting each antenna element a single radio frequency (RF)signal [9]. Moreover, the codebook has several ways to set antenna weight coefficients. Training is the way to find the best beam pair between transmitter and receiver [10]. Taking IEEE802.15.3c as a reference codebook, there are many studies conducted in different ways to construct codebooks. In [10], uniform weighting based codebook is designed by simple weighting window function and a weighting vector constituting a conjugate number. The circular antenna codebook weight vector is constructed by circularly shifting each column [1].

In this paper, we propose a new form of codebook design based on the number of phase states. Additionally, we compare the proposed codebook with the reference codebooks using SNR and AF as performance evaluation factors. Furthermore, we demonstrate the effect of the number of antenna elements on the generated beams, and how to make the beam wide or narrow, as well as the compressing the beam to cover narrow-angle with high gain and directivity.Weprovide some results and conclusions about the codebooks design and its evaluation, regarding AF and SNR. In addition, we demonstrate the effect of the number of antenna element on the generated beam.

\section{System model}

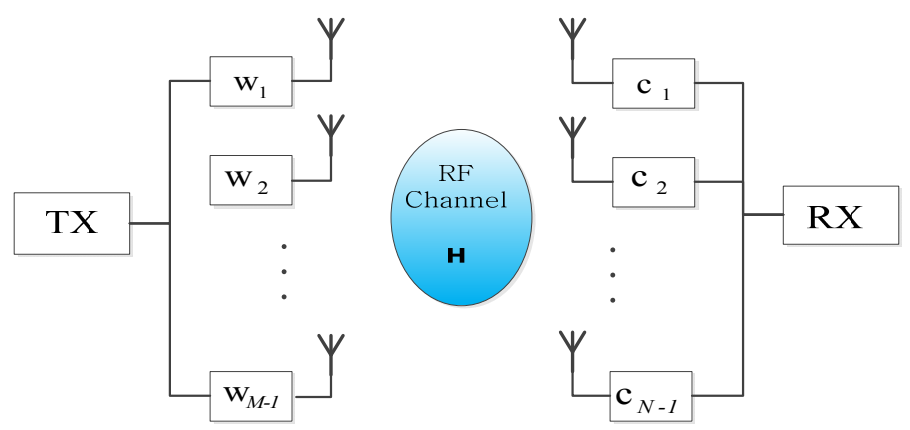

Fig. 1. MIMO System Model

The MIMO system model is shown in Figure 1 The device 1 (DEV1) is equipped with a linear array of $M$ transmit antennas, the device 2 (DEV2) has $N$ receive antenna. Data transmission between the two devices involves the following: at the transmitter side, the signal after baseband processing is upconverted to RF band, then multiplied by transmitter code vector $\mathrm{w}$. On the receiver side, then the received RF signal is down-converted to abaseband signal, and then multiplied by receiver code vector c; both weight vectors satisfy 
$\left|w_{i}\right|=\left|c_{i}\right|=1$.We can generate beams by shifting each antenna element RF signal, each column (beam) is circularly generated by shifting a previous beam by angle $2 \pi / N[1]$, [7].

\subsection{Codebook design and principles}

The followings are fundamental codebooks design principles [11]: First, the codebook designed for the phased antenna, each column of codebook matrix determine the phase shift of each element. Second, the IEEE802.15.3c codebook constructed with $\pi / 2$ phase resolution without changing in amplitude. Third, codebooks support different antenna configurations and available for multi-antenna elements. Fourth, the columns in the codebook are orthogonal. Therefore multiple beams can be generated simultaneously with minimum interference [3], [10].

\subsection{IEEE802.15.3c codebook design}

Let's consider a linear array antenna with uniform spacing of half wavelength, the system with $\mathrm{M}$ antenna elements and $K$ is the required number ofbeam patterns generated by the codebook, then when $K \geq M$, each element of the codebook matrix is given by:

$$
w(m, k)=j^{f i x\left(\frac{m \times \bmod (k+(k / 2), K)}{K / 4}\right)} .
$$

where $m=0,1, \ldots, M-1, k=0,1, \ldots, K-1$, also the same definition for all codebooks.

The function $\operatorname{fix}(x)$ returns the biggest integer, which is smaller or equal to its value, this codebook is called IEEE 802.15.3c codebook with four discrete phases [10]. From (1) we can express N-phase's codebook as follow:

$$
w(m, k)=e^{j \frac{2 \pi}{N} f i x(m \times \bmod (k+(K / 2), K))} .
$$

where $N$ indicates the number of discrete phases.

\subsection{Uniform weighting based codebook}

Uniform weighting is a simple window weighting function scheme; the weights are selected to generate beam patterns in a given directions according to the direction of main response axis. When the main response axis (MRA) in the direction of $\theta_{k}$, where $\theta_{k}$ is the direction ofthe $k^{\text {th }}$ beam, if the normal-line is 1-D array antenna, and the uniform antenna element spacing is $d(d=\lambda / 2)$, then the codebook weighting vector elements is given by:

$$
w(m, k)=e^{j m \pi d \cdot \sin \left(\theta_{k}\right)}
$$

\subsection{Proposed codebook}

The 2-bit discrete resolution codebook, for $M$ antenna elements of theuniform phasearray, is similar as described in (1), this codebook matrix each column is corresponding to the phase rotation of antenna elements. The 2-bit resolution codebook in (1) generates patterns with different gain along the main axis because 2-bit resolution limits the phase 
quantization, it is clear that the beam patterns generated by (1) as mentioned in[10] have anunequal gain. We propose a different codebook weighting element construction, compared with other reviewed codebooks, the main difference is the phase state, which follows $\varphi=2^{n}$, where $n$ is integer positive number. The expression of the entire codebook weighting elements is given as:

$$
w(m, k)=j^{\left(\frac{(m-1)(k-1)-K / 2}{\varphi / 4}\right)} .
$$

The $n$-bit codebook as expressed in (4) is reasonable to improve the phase resolution and achieves equal gain along the main response axis.

The codebooks in (1), (3), and (4) consider as auniform spacing linear array antenna. Therefore 1-D AF can be written as (5):

$$
A F_{k}(\theta)=\sum_{m=0}^{M-1} w(m, k) e^{j \frac{2 \pi m d}{\lambda} \cos (\theta)} .
$$

The transmitter weight vector can bewritten as $\mathrm{w}_{k}=\left[w_{0} w_{1} \ldots w_{M-1}\right]^{\mathrm{T}}$, where $\theta$ is thepolar angle of the direction of arrival (DOA), $d$ is the spacing between elements; $\lambda, K$, and $M$ are signal wavelength, required beam patterns and a number of antenna elements respectively [4].Similarly, we can obtain 2-D AF and codebook weight vector from corresponding 1-D $\mathrm{AF}$ and codebook in a given direction. Assume, we have $M$ number of antenna elements are located in an $x-y$ plane. Then the polar angle in the $x$-direction is $\theta$, the azimuth angle in the $z$-direction is $\phi$. Similarly, $d_{x}$ and $d_{y}$ are the spacing between elements. Moreover, $M_{x}$ and $M_{y}$ represent the number of antenna elements in agiven directions.

Therefore, the2-DAF resulting in AF in $x$-direction multiplied by AF in they-direction as: $A F(\theta, \phi)=A F_{x}(\theta, \phi) * A F_{y}(\theta, \phi)$, by using the summation we will have [11]:

$$
A F(\theta, \phi)=\sum_{m_{1}}^{M_{x}-1} \sum_{m_{2}}^{M_{y}-1} w_{m_{1}, m_{2}} e^{j 2 \pi\left[\frac{m_{1} d_{x}}{\lambda} \cos \theta \sin \phi+\frac{m_{2} d_{y}}{\lambda} \sin \theta \sin \phi\right]} .
$$

\subsection{Circular antenna codebook design}

Suppose the circular array antenna consists of two layers, the outer circle with radius $r_{1}\left(r_{1}=M \lambda / 16\right)$, where $M$ is the number of antenna elements in multiple of 8 , the inner circle radius is $r_{2}\left(r_{2}=\lambda / 2\right)$, for more information about inner and outer layers referredin [1]. In this study, we focus on the outer circle to design codebook of circular array antenna which is used mainly to produce the beam patterns, the weight vector for this codebook as follows:

$$
w(m, k)=e^{-j 2 \pi \frac{r_{1}}{\lambda} \cos \left(\frac{2 \pi \times \bmod ((m-k), M)}{M}\right)} .
$$

1-D Antenna array response of circular codebook is given by:

$$
A F_{k}(\theta)=\sum_{m=0}^{M-1} w(m, k) e^{j 2 \pi \frac{r}{\lambda} \cos \left(\theta-\frac{2 \pi m}{M}\right)}
$$


Similarly, 2-D array response expression as follow:

$$
A F_{k}(\theta, \phi)=\sum_{m=0}^{M-1} w(m, k) e^{j 2 \pi \frac{r}{\lambda}\left[\sin \theta \cos \left(\phi-\phi_{m}\right)-\sin \theta_{0} \cos \left(\phi_{0}-\phi_{m}\right)\right]}
$$

We can rewrite(9) as:

$$
A F_{k}(\theta)=\sum_{m=0}^{M-1} w(m, k) e^{j\left[2 \pi \frac{r}{\lambda} \sin \theta \cos \left(\phi_{0}-\phi_{m}\right)+\alpha_{m}\right]} .
$$

The maximum AF occurs when all phases equal to unity or $2 \pi \lambda^{-1} r \sin \theta \cdot \cos \left(\phi-\phi_{m}\right)+\alpha_{m}=2 \pi i$ where $i=0, \pm 1, \pm 2 ; \forall m$; the principal of maximum pattern direction when $i=0$ is defined by $\left(\theta_{0}, \phi_{0}\right)$, where $\alpha_{m}=-2 \pi \frac{r}{\lambda} \sin \theta \cos \left(\phi-\phi_{m}\right), \phi_{m}=\frac{2 \pi m}{M}$.

\subsection{SNR expression}

At the beginning of transmission, the RF signals weighted at DEV1 by applying weighting vector $\mathrm{w}=\left[\begin{array}{llll}w_{1} & w_{2} & \ldots & w_{N_{t}}\end{array}\right]^{\mathrm{T}}$ at the transmitter side. Then the $N_{t} \times 1$ signal vector will be produced and transmitted. The transmitted signal will be received by DEV2, by applying receiver weight vector $\mathrm{c}=\left[\begin{array}{llll}c_{1} & c_{2} & \ldots & c_{N_{r}}\end{array}\right]^{\mathrm{T}}$, which is predefined code vector at the receiver side, the combined received signal and noise in [13] as:

$$
\mathbf{r}=\mathrm{c}^{\mathrm{H}} \mathbf{H} \mathbf{w} \mathbf{s}+\mathrm{c}^{\mathrm{H}} \mathbf{n}
$$

where $\mathrm{c}^{\mathrm{H}}$ is conjugate transpose matrix of DEV2codebook vector c, $\mathbf{H}$ is $N_{t} \times N_{r}$ channel matrix which constructed as in [14], $\mathbf{s}$ is $N_{t} \times 1$ transmitted signal and $\mathbf{n}$ is $N_{r} \times 1$ additive white Gaussian noise with variance $\sigma$. The normalizedtotal power of all transmit antennas is:

$$
\mathrm{E}\left[|\mathbf{s}|^{2}\right]=\frac{1}{N_{t}}
$$

The weight vector follows the relation: $\mathrm{w}^{\mathrm{H}} \mathrm{W}=N_{t}$ and $\mathrm{c}^{\mathrm{H}} \mathrm{c}=N_{r}$, from (11), the output SNR can be derived as:

$$
\gamma=\mathrm{E}\left[\left|\mathrm{c}^{\mathrm{H}} \mathbf{H} \mathrm{ws}\right|^{2}\right] / \mathrm{E}\left[\left|\mathrm{c}^{\mathrm{H}} \mathbf{n}\right|^{2}\right]
$$

Consequently, from (12) the expression in (13) can be rewritten as:

$$
\begin{aligned}
\gamma & =\mathrm{E}\left[\left|\mathrm{c}^{\mathrm{H}} \mathbf{H w}\right|^{2}\right] * \mathrm{E}\left[|\mathbf{s}|^{2}\right] / N_{r} \sigma^{2} . \\
& =\left|\mathrm{c}^{\mathrm{H}} \mathbf{H w}\right|^{2} / N_{t} N_{r} \sigma^{2}
\end{aligned} .
$$

Expression (14) explains the received SNR. 


\section{Results and discussion}

In this section, we evaluate the performance of the proposed codebook with the reference codebooks through simulation.

\subsection{SNR Evaluation}

We obtainthe results by assuming the number of beams $K=64$, and we calculate the SNR at different noise variances. In Figure 2(a), we suppose the noise variance $\sigma=0.5$, the proposed codebook achieves a higher dynamic range of SNR than the conventional codebooks, almost 30dB. Although, in some codebook indices the proposed codebook gets the highest SNR among the rest of the codebooks. The main reason behind these variations is due to the geometric arrangement of the weight elements, which mainly depend on the steering weighting function of each codebook.

By taking $\sigma=1$, as shown in Figure 2(b), the comparison trends look the same as $\sigma=0.5$. However, the effect of noise dramatically degrades the absolute value of SNR for all codebooks by about $15 \mathrm{~dB}$. Itis verified in equation (14), in which more noise is gained by the factor number of receiver antenna elements. The results show that the proposed codebook has a higher peak and dynamic range than the reference codebooks.In Figures 3 and 4, we obtain the CDF and PDF of SNR respectively. From Figure 3, the SNR distribution of the uniform codebook is very narrow compared with the other three codebooks because this codebook has a small dynamic range. On the other hand, the probability of CDF of the proposed codebook has a higher value than other codebooks with higher dynamic range. From Figure4, the proposed codebook and the IEEE802.3c have wide SNR and comparable PDF values, unlike the uniform and circular codebooks which have narrow SNR values.

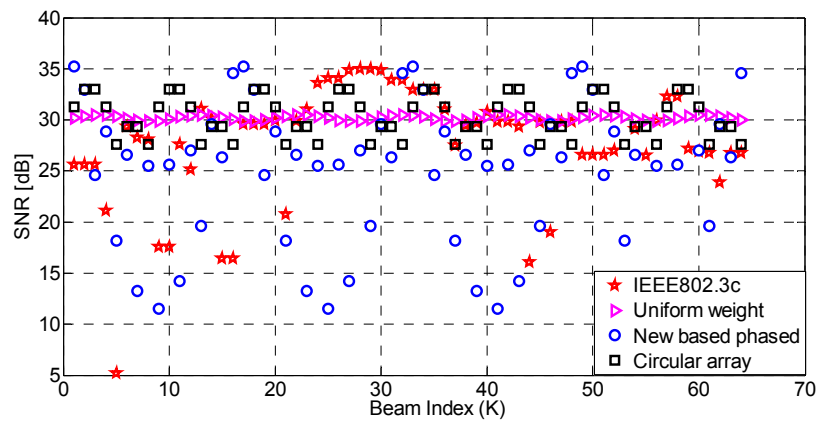

(a)

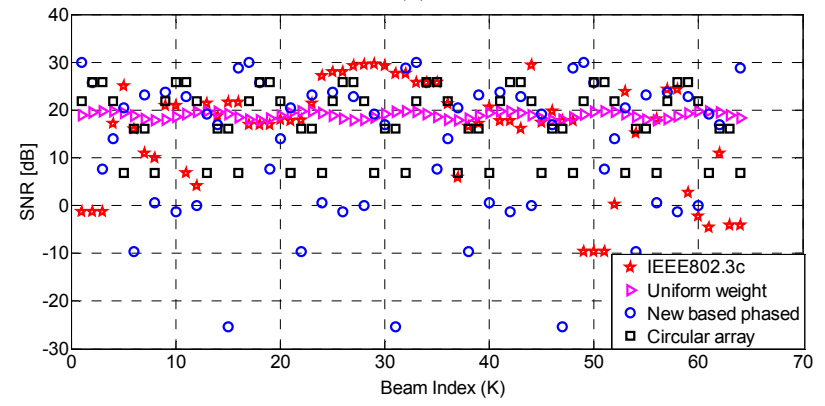

(b)

Fig. 2. SNR versus beam index: (a) $\sigma=0.5$; (b) $\sigma=1$ 


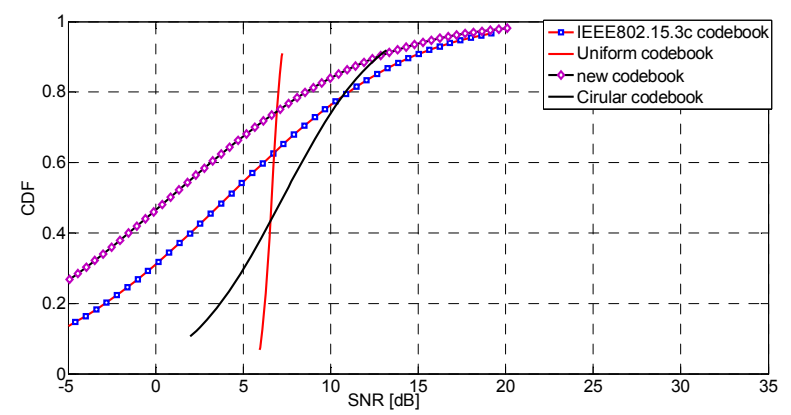

Fig.3. CDF for SNR of codebooks with respect to $\sigma=0.5$

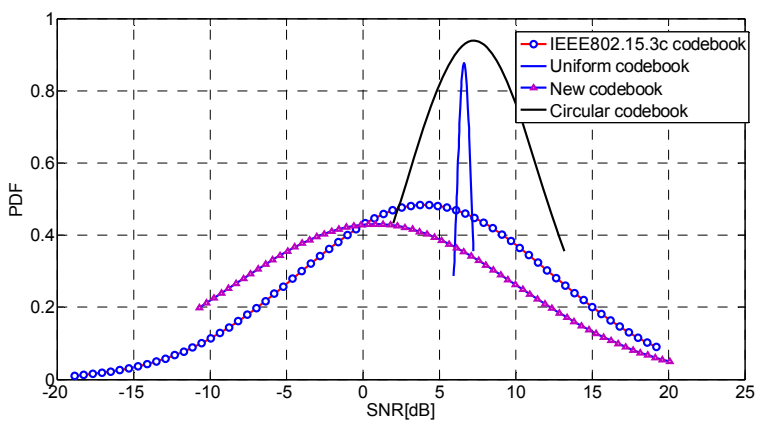

(b)

Fig. 4. PDF for SNR of codebooks: with respect to $\sigma=1$

\subsection{Array factor evaluation}

In this paper, we compute $\mathrm{AF}$ as a function of 2-D angles to get the 3-D plot as shown in Figures5, 6, 7, and 8. Elevation and azimuth angles are considered for linear array antenna in the range $[0, \pi]$, and for circular array antenna in the range $[-\pi, \pi]$. By increasing with selected step angle $\alpha$, i.e. $[\theta+\alpha, \phi+\alpha]$, the proposed and the IEEE802.15.3c codebooks have maximum responses from $(0, \pi / 4)$ to $(5 \pi / 6, \pi)$ as shown in Figure5 and Figure 6. However, IEEE802.15.3c has more peak sidelobes than the proposed codebook. In Figure7 the uniform codebook has maximum AF at $(\pi / 2, \pi / 2)$ with small side lobes at $(\pi / 2, \pi / 4)$ and $(\pi / 2,3 \pi / 2)$. From Figure8, the circular codebook has maximum values at $(\pi,-\pi / 2)$ and $(-\pi, \pi)$ with small minor side lobes. Thus, in terms of coverage, the circular codebook is desirable. On the other hand, regarding theratio of peak to minor side lobes, the proposed codebook is the choice. However, the ratio of peak-to-side lobes gives more emphasis than the coverage in $\mathrm{mmW}$ communication. Hence, the proposed codebook is preferably better than others. 


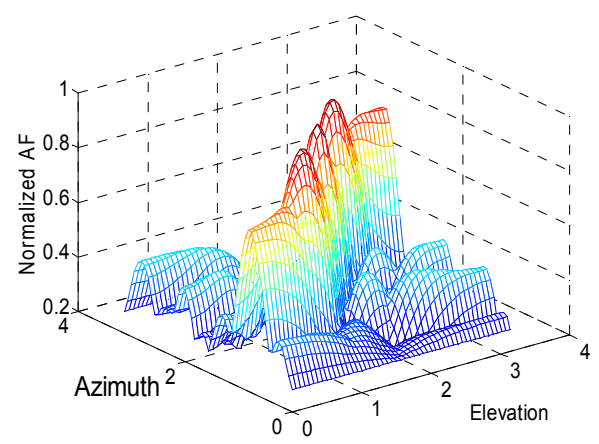

Fig. 5.3-D Array Factor for IEEE802.3c codebook

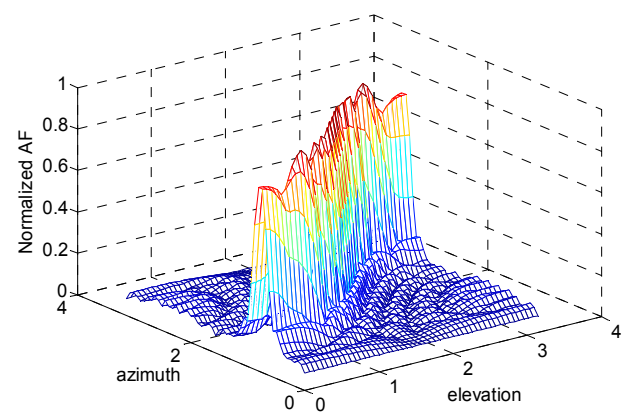

Fig. 6.3-D Array Factor for the proposed codebook

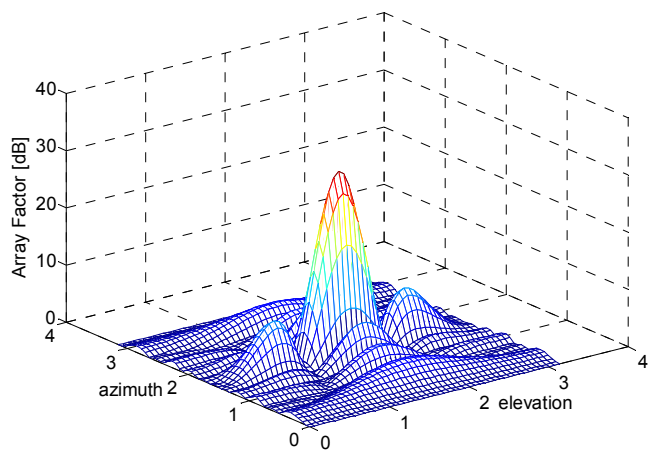

Fig. 7.3-D Array Factor for the Uniform weight codebook

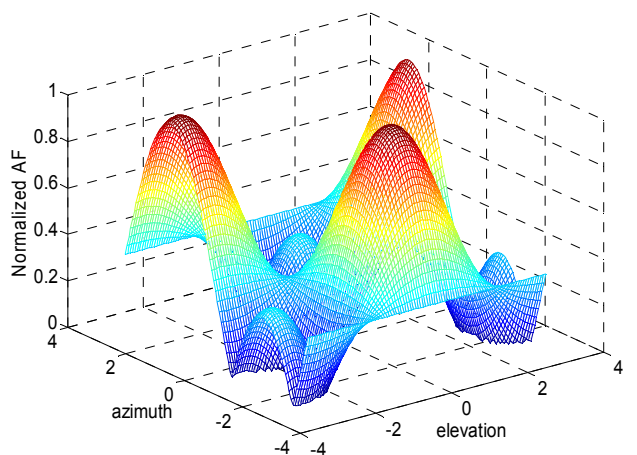

(d)

Fig. 8.3-D Array Factor for Circular array codebook 


\subsection{Effect of the number of antenna elements}

The number of antenna elements hasan excellent contribution on the designed beams as shown in Figures9, 10, 11, and 12. The beam width of each generated and the beam-gain fully depends on the number of antenna elements, as illustrated in Figure9 the number of antenna elements $(M)$ equal to 32 and the number of beams $(K)$ equal to 16 . The beams interferewith each other, and the beam width is wide relatively. Consequently, Figure10 $M=100$ and $K=16$ with a narrow beam and reduces the beams interfere. Refer to the results in Figure11 and Figure12. Meanwhile, by increasing the number of antenna elements, we can cover the area of all direction (Omni-direction) without beams interference.

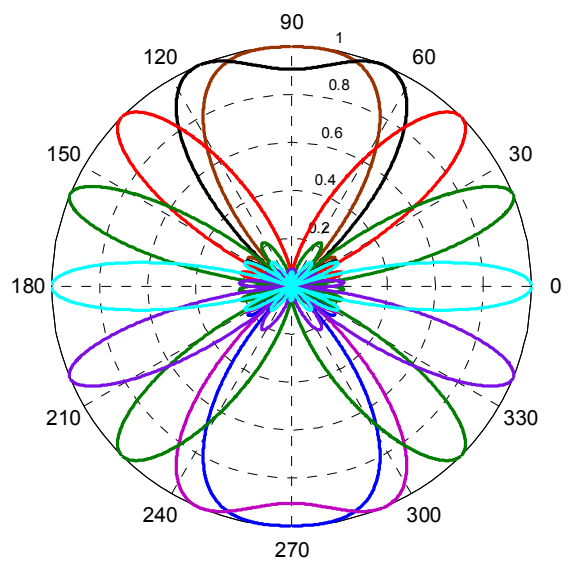

Fig. 9. Effects of the number of antenna elements on the generated beams when $M=32$ and $K=16$

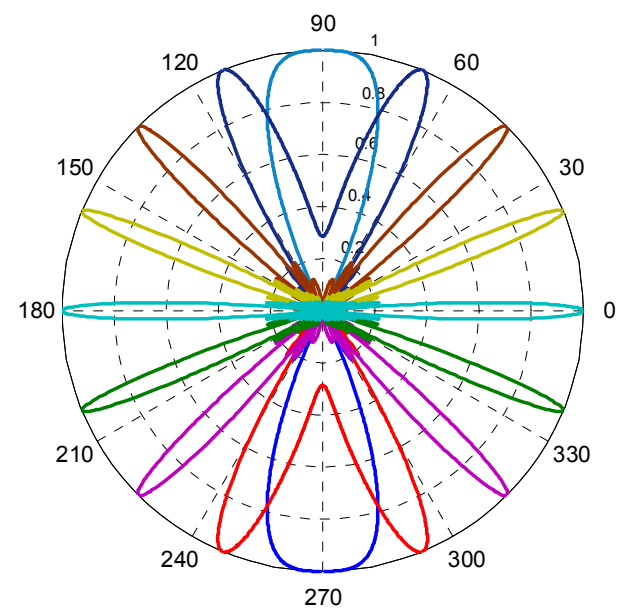

Fig. 10.Effects of the number of antenna elements on the generated beams when $M=100$ and $K=16$ 


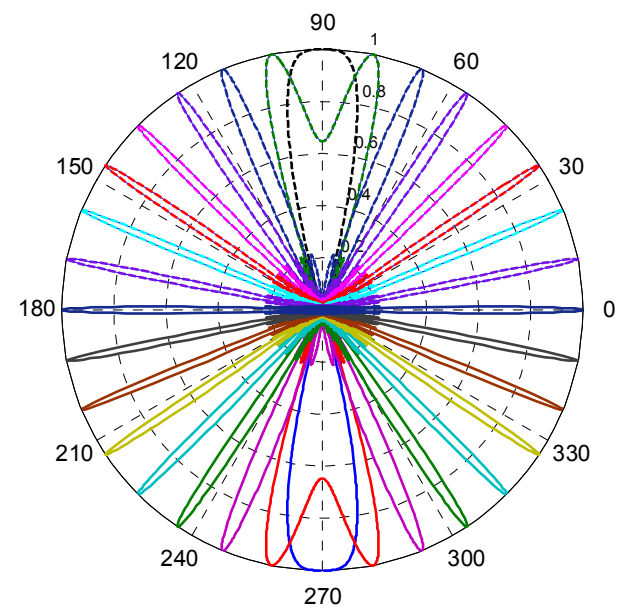

Fig. 11.Effects of the number of antenna elements on the generated beams when $M=256$ and $K=32$

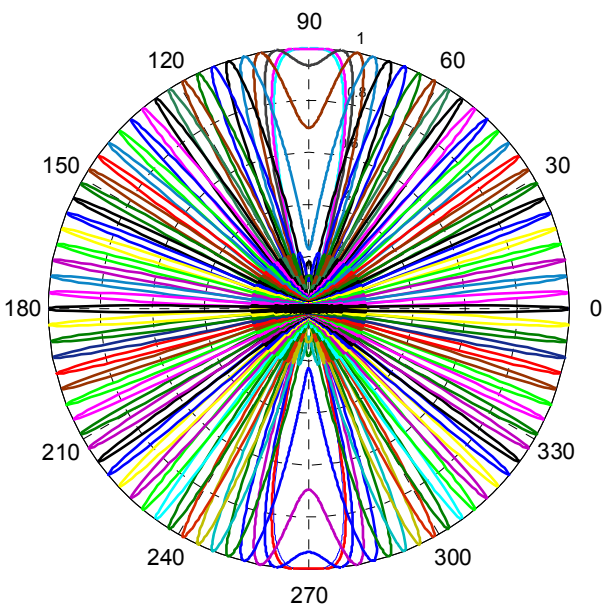

(d)

Fig. 12.Effects of the number of antenna elements on the generated beams when $M=256$ and $K=100$

\section{Conclusion}

In this paper, a new codebook is proposed and compared with the IEEE 802.15.3c, uniform weighting, and circular array antenna codebooks. The results show that the proposed codebook achieves better SNR, array directivity as well as small side lobes, compared with the IEEE 802.15.3c, but higher peak and wider dynamic range of SNR and better array directivity compared with the uniform weighting and circular antenna codebooks. Generally, the proposed codebook has much smaller side lobes and wide main response angle regarding the conventional codebooks. Thus, the proposed codebook is expected to improve signal quality and increase user throughput significantly.

Acknowledgment: This work was supported by National Key Laboratory of Electromagnetic Environment, China Research Institute of Radiowave Propagation under grant No. 201600012 


\section{References}

1. Feng, W., Xiao, Z., Jin, D., Zeng, L.: Circular-antenna-array-based codebook design and training method for $60 \mathrm{GHz}$ beamforming. IEEE Wirel. Commun. Netw. Conf. WCNC, pp. 4140--4145 (2013).

2. Wang, J.: Beam codebook based beamforming protocol for multi-Gbps millimeterwave WPAN systems. IEEE J. Sel. Areas Commun, vol. 27, no. 8, pp. 1390--1399 (2009)

3. Xiao, Z., He, T., Xia, P., Xia, X.: Hierarchical Codebook Design for Beamforming Training in Millimeter-Wave Communication. IEEE Trans. Wireless Commun, vol. 15, no. 5, pp. 3380--3392 (2016)

4. Kutty, S.,Sen, D.: An improved numerical optimization method for efficient beam search in $60 \mathrm{GHz}$ indoor millimeter wave wireless networks. Int. Symp. Adv. Networks Telecommun. Syst. ANTS, vol. 2016-Febru, pp. 1--6 (2016)

5. Lee, H., Ko, Y.: Low Complexity Codebook-Based Beamforming for MIMO-OFDM Systems in Millimeter-Wave WPAN. IEEE Trans. Wireless Commun, vol. 10, no. 11, pp. 3607--3612 (2011)

6. Zou, W., Wang, L., Guo, C.: 3D-beamforming codebook design based on directly search for millimeter-wave communications. Proc. - IEEE INFOCOM, vol. 2016Septe, pp. 1003--1008 (2016)

7. Hosoya, K., Prasad, N., Ramachandran, K., Orihashi, N., Kishimoto, S., Rangarajan, S., Maruhashi, K.: Multiple sector ID capture (MIDC): A novel beamforming technique for $60-\mathrm{GHz}$ band multi-Gbps WLAN/PAN systems. IEEE Trans. Antennas Propag., vol. 63, no. 1, pp. 81--86 (2015)

8. Harry, L., Trees, V.: Optimum Array Processing. Part IV of Detection, Estimation, and Modulation Theory, (2002)

9. Weixia, Z., Chao, G., Guanglong, D., Zhenyu, W., Ying, G.: A new codebook design scheme for fast beam searching in millimeter-wave communications. vol. 11, no. 6, pp. $12--22(2014)$

10. Zou, W., Cui, Z., Li, B., Zhou, Z., Hu, Y.: Beamforming codebook design and performance evaluation for $60 \mathrm{GHz}$ wireless communication," 11th Int. Symp. Commun. Inf. Technol. Isc. 2011, no. Iscit, pp. 30--35 (2011)

11. Wang, J., Lan, Z., Sum, C., Pyo, C., Gao, J., Baykas, T., Rahman, A., Funada, R., Kojima, F., Lakkis, I., Harada, H., Kato, S.: Beamforming codebook design and performance evaluation for $60 \mathrm{GHz}$ wideband WPANs. IEEE Veh. Technol. Conf, pp. $0--5$ (2009)

12. Zou, W., Du, G., Li, B., Zhou, Z.: A unified codebook for fast beam searching in millimeter-wave communications. 2012 Int. Conf. Comput. Probl. ICCP 2012, no. 61171104, pp. 218--223 (2012)

13. Zhang, H., Wu, C., Cui, X., Gulliver, T., A., Zhang, H.: Low complexity codebookbased beam switching for $60 \mathrm{GHz}$ anti-blockage communication. J. Commun., vol. 8, no. 7, pp. 428--439 (2013)

14. He, T., Xiao, Z.: Suboptimal Beam Search Algorithm and Codebook Design for Millimeter-Wave Communications. Mob. Networks Appl., vol. 20, no. 1, pp. 86--97, (2015) 